\title{
Induction of Tolerance to Fusarium Wilt and Defense-Related Mechanisms in the Plantlets of Susceptible Berangan Banana Pre-Inoculated with Pseudomonas sp. \\ (UPMP3) and Burkholderia sp. (UPMB3)
}

\begin{abstract}
This study is aimed at assessing the ability of two endophytic bacteria originally isolated from healthy oil palm roots, Pseudomonas sp. (UPMP3) and Burkholderia sp. (UPMB3) to induce resistance in susceptible Berangan banana against Fusarium oxysporum race 4 (FocR4). Increased accumulation of resistance-related enzymes such as peroxidase (PO), phenylalanine ammonia lyase (PAL), lignithioglycolic acid (LTGA), and pathogenesisrelated (PR) proteins (chitinase and $\beta$-1,3-glucanase) has been observed in plantlets treated with endophytic bacteria UPMP3 and UPMB3 singly or as mixture under glasshouse conditions. Pre-inoculation of banana plantlets with UPMP3 showed a significant reduction in Fusarium wilt incidence $72 \mathrm{~d}$ after challenged inoculation with FocR4. UPMB3 was less effective in suppressing Fusarium wilt compared to UPMP3, whereas, the mixture of both endophytes showed an intermediate effect. Based on these results, it is concluded that UPMP3 could be a promising biological control agent that can trigger resistance against Fusarium wilt in susceptible Berangan banana.
\end{abstract}

Keyword: banana, Pseudomonas sp., Burkholderia sp., induce resistance, Fusarium wilt 